\title{
Contribuições de um treino cognitivo para a memória de idosos institucionalizados
}

\author{
Isabelle Patriciá Freitas Chariglione - Universidade de Brasília, Brasília, Brasil \\ Gerson Américo Janczura - Universidade de Brasília, Brasília, Brasil
}

\begin{abstract}
Resumo
Este estudo investigou a influência de diferentes treinos cognitivos na memória de idosos institucionalizados e em medidas neuropsicológicas e de humor. Dezesseis idosos participaram do programa que incluiu dois tipos de treinamentos que variavam quanto à semelhança com as atividades e o ambiente dos idosos na instituição. Os treinos tinham a duração de 60 minutos e ocorreram duas vezes por semana durante quase quatro semanas. Os resultados mostraram que ARFC foi influenciado pelo tipo de treinamento, e que a recordação livre para palavras foi melhor após o treinamento somente para o grupo de idosos alfabetizados, independentemente do tipo de treinamento. Não foram detectados efeitos no teste de reconhecimento de imagem e as sessões de treinamento afetaram positivamente os escores EDG.
\end{abstract}

Palavras-chave: Treino cognitivo, Idosos institucionalizados, Avaliação neuropsicológica, Recordação, Reconhecimento.

Contributions of a Cognitive Training for the Memory of Institutionalized Elderly

\begin{abstract}
This study investigated the influence of different cognitive training procedures in memory, neuropsychological measures and mood of institutionalized elderly. Sixteen seniors attended the program which included two types of procedures that varied in terms of the similarity to the daily activities and environment of the institutionalized elderly. The training lasted 60 minutes and occurred twice a week for almost four weeks. The results showed that ARFC was influenced by the type of training; free recall for words was better after training only for literate individuals, regardless of training type. No positive effects were detected in picture recognition. Training sessions had a positive influence in GDS scores. Keywords: Cognitive training Institutionalized elderly, Neuropsychological assessment, Recall, Recognition.
\end{abstract}

\section{Contribuciones de un entreno cognitivo para la memoria de ancianos institucionalizados}

\begin{abstract}
Resumen
Este estudio investigó la influencia de diferentes entrenos cognitivos en la memoria de ancianos institucionalizados y en medidas neuropsicológicas y del humor. Dieciséis ancianos participaron del programa que incluyó dos tipos de entrenamientos que variaban cuanto a la semejanza con las actividades y el ambiente de ancianos en la institución. Los entrenos tenían la duración de 60 minutos y ocurrieron dos veces por semana durante casi cuatro semanas. Los resultados mostraron que ARFC fue influenciado por el tipo de entrenamiento, y que el recuerdo libre para palabras fue mejor pasado el entrenamiento solamente para el grupo de ancianos alfabetizados, independientemente del tipo de entrenamiento. No fueron detectados efectos en el teste de reconocimiento de imagen y las sesiones de entrenamiento afectaron positivamente los escores EDG.

Palabras-clave: Entreno cognitivo, Ancianos institucionalizados, Evaluación neuropsicológica, Recuerdo, Reconocimiento.
\end{abstract}

O envelhecimento da população é uma realidade, sendo, a faixa etária a partir de 60 anos de idade, a que mais cresce (Regi, Junior \& Castro, 2007). Estima-se que, em 2025, haverá cerca de 32 milhões de idosos no Brasil. As demências, caracterizadas pela perda das habilidades cognitivas e emocionais, são consideradas um problema de Saúde Pública que interfere na vida diária dos idosos, despertando, em consequência, mais interesse pela pesquisa neste campo.

O comprometimento da memória é um dos principais temas de pesquisa sobre o processo de envelhecimento. Pouca atenção tem sido dispensada à compreensão e investigação da memória em um contexto particular, frequentemente vivenciado pelos idosos e seus familiares, que se relaciona aos idosos em instituições de longa permanência. Nesses ambientes é cada vez mais importante a capacitação de profissionais com enfoque não apenas momentâneo, mas que se promova um processo contínuo de atendimento integral e qualificado à saúde do idoso.
Pesquisadores da longevidade humana (p. ex., Pavarini, Mediondo, Barham, Varoto \& Filizola, 2005) explicam que o aumento da expectativa de vida tem coincidido com uma concentração da longevidade média. A curva de sobrevivência está tomando uma forma que indica o aumento da expectativa de vida dessa população.

O estudo do envelhecimento cognitivo humano vem progredindo significativamente nos últimos 50 anos (Yassuda, Batistoni, Fortes \& Neri, 2006). Há consenso, atualmente, que determinados déficits de memória também caracterizam o envelhecimento saudável e natural do ser humano. Essa afirmação se apoia em estudos que detectaram diferenças entre adultos jovens e idosos em tarefas envolvendo a memória operacional e a memória episódica (StuartHamilton, 2002; Yassuda e cols., 2006); pesquisas relacionadas aos processos mnésicos que encontraram diferenças significativas entre jovens e idosos durante a codificação e o resgate das informações (Kausler, 
1991); e, ainda, evidências que apontam para uma lentidão no processamento das informações em idosos (Salthouse, 1991).

\section{O treino cognitivo na terceira idade}

A reabilitação, ou a busca por melhoras em determinadas funções cognitivas, teve um início provável na Alemanha, durante a Primeira Guerra Mundial, com o objetivo de auxiliar a memória de soldados com lesões cerebrais. Durante a Segunda Guerra Mundial, Luria (1990) teve um papel importante na reabilitação neuropsicológica, pois foi o responsável pela organização de um hospital para soldados com aquela condição neurológica.

A reabilitação tem por fim melhorar a qualidade de vida dos pacientes e familiares, maximizando o aproveitamento das funções total ou parcialmente preservadas por meio do ensino de "estratégias compensatórias, aquisição de novas habilidades e a adaptação às perdas permanentes" (D'Almeida, Pinna, Martins, Siebra \& Moura, 2004, p. 4). O processo de reabilitação proporciona uma conscientização do paciente a respeito de suas capacidades remanescentes, produzindo uma mudança na auto-observação e, possivelmente, uma aceitação de sua nova realidade.

Wilson (2009) diferencia a reabilitação cognitiva da reabilitação neuropsicológica. A reabilitação cognitiva visa capacitar pacientes e familiares a conviver, diminuir e ou superar as deficiências cognitivas derivadas de lesões neurológicas, mas focase, principalmente, na melhora das funções cognitivas administrando treinos cognitivos. Já a reabilitação neuropsicológica trata dos déficits cognitivos e das alterações emocionais e comportamentais, melhorando a qualidade de vida do paciente.

Muitos estudos indicam que intervenções complexas envolvendo técnicas de memorização, relaxamento e atenção podem gerar efeitos positivos e duradouros em idosos, especialmente quando empregadas em grupo (Yassuda, 2002). Segundo Guerreiro e Caldas (2001), diversos pesquisadores encontraram uma relação positiva entre o desempenho cognitivo do idoso e uma estimulação continuada. O exercício diário da mente promoveria a vivacidade mental e as atividades promotoras de estimulação mental contribuiriam, ainda, para a prevenção do declínio cognitivo.

Neely (2000) ressalta que um dos maiores ganhos da aplicação de pesquisas sobre o treino de memória é entender a complexidade de fatores relacionados ao aprimoramento da memória, a fim de se desenvolver uma intervenção eficiente nesse processo cognitivo. A autora prevê o aprimoramento da memória em função de vários fatores críticos, como, por exemplo, as diferenças individuais, questões metodológicas e as habilidades e processos treinados, e chama a atenção para as habilidades e processos que deveriam ser focalizados a fim de atingir este objetivo. Ela sugere que para assegurar os benefícios positivos em intervenções de memória é necessário direcionar o treino a tarefas particulares, habilidades e comportamentos que são de interesse primário do aprendiz.

Diversos estudos (p. ex., Belleville, 2008; Belleville, Gilbert, Fontaine, Gagnon, Ménard, \& Gauthier, 2006; Belleville, Clément, Mellah, Gilbert, Fontaine \& Gauthier, 2011; Gagnon \& Belleville, 2012; Gross, Rebok, Unverzagt \& Brandt, 2011) têm corroborado a hipótese de que mudanças, tanto cognitivas quanto neurobiológicas, ocorrem em idosos (independente da faixa etária) que utilizam treino cognitivo, incluindo idosos saudáveis, normais e aqueles portadores de alguma classe específica de patologia cognitiva.

No que se refere especificamente a aspectos de memória, Belleville e cols. (2006) demonstraram que pacientes canadenses com comprometimento cognitivo leve (CCL) conseguiram melhorar suas performances em memória episódica quando submetidos a um treino de memória multifatorial. Resultados semelhantes foram verificados na Alemanha por Kurz, Pohl, Ramsenthaler e Sorg (2008) com o mesmo tipo de população tendo coletado, além de medidas de memória, medidas de humor e de atividades de vida diária que evidenciaram alterações positivas e significativas nessas avaliações. $\mathrm{Na}$ população brasileira, Irigaray, Schneider e Gomes (2011) obtiveram resultados semelhantes em avaliações de desempenhos cognitivos, qualidade de vida e de bem-estar psicológico.

Além dessas questões, há uma preocupação relacionada à escolaridade, ao envelhecimento e à cognição, pois, especificamente no caso dos idosos institucionalizados, verificam-se poucos estudos sobre o seu padrão de desempenho em avaliações neuropsicológicas (p. ex., Foss, Vale \& Speciali, 2005). Os testes envolvidos nas avaliações neuropsicológicas representam uma tarefa incomum para grupos de diferentes culturas ou analfabetos, assim como treinos que possam produzir benefícios a essa população.

Para a execução do treino desenvolvido neste trabalho foram desenvolvidas duas versões, sendo uma mais relacionada à rotina do idoso e outra menos relacionada à sua rotina. Uma análise do ambiente foi realizada para orientar que elementos estariam mais e menos relacionados à rotina desse idoso. Esses elementos se referiam ao ambiente físico da instituição, atividades realizadas pelos idosos (jogos/hobbies), alimentos disponíveis nas refeições, objetos pessoais 
encontrados na instituição, além de pessoas e profissões a que eles tinham acesso naquele local.

Então, com base nessas informações e em aspectos relacionados ao funcionamento da memória, elaboramos treinos específicos que, de forma extensiva, multifatorial e baseado em antigas e novas tecnologias, facilitassem o exercício cognitivo dos idosos - como será detalhado na seção método do presente artigo.

\section{Avaliando os ganhos cognitivos}

Os testes neuropsicológicos aplicados, especificamente, na população idosa devem ser breves, simples e de fácil administração, para que atinjam o seu principal objetivo, que é servir como instrumento rápido de avaliação, triagem e estratificação de risco, e para que possam ser aplicados em qualquer unidade básica de saúde. A escolha de um ou outro teste dependerá da familiaridade que o examinador tenha com sua interpretação (Lezak, 1995).

Atualmente, um dos testes mais aplicados na avaliação neuropsicológica é o Mini Mental State - MMS (Folstein, Folstein \& McHugh, 1975), que avalia orientação, aprendizagem, controle mental, denominação, repetição, compreensão de uma ordem tripla e a cópia de um desenho. Outro instrumento é o teste de Avaliação Rápida das Funções Cognitivas ARFC (Gil, 2005; Gil, Toullat \& Pluchon, 1986), que tem uma correlação alta e significativa com o MMS e permite que se faça, em menos de 15 minutos (dependendo das limitações apresentadas pelo indivíduo a ser examinado), um miniexame neuropsicológico verificando a orientação, aprendizagem, memória imediata, cálculo mental, raciocínio e julgamento, compreensão, denominação, repetição, compreensão de uma forma escrita, fluidez verbal, praxias ideomotora e construtiva, identificação de um desenho e a escrita (Gil, 2005).

Além de avaliar a capacidade cognitiva, é necessário identificar a ocorrência de comorbidades, como doenças cerebrais, demências e depressão, pois algumas das queixas de perda de memória estão associadas a quadros depressivos, nos quais as capacidades intelectuais do idoso encontram-se prejudicadas (Almeida \& Forlenza, 1997). A fim de verificar a existência de um eventual distúrbio de personalidade e ou de estado depressivo pode-se recorrer à Escala de Depressão Montgomery e AsbergMADRS (Montgomery \& Asberg, 1979), assim como à Escala de Depressão Geriátrica EDG (Almeida \& Almeida, 1999; Sheikh \& Yesavage, 1986).

A eficácia do treino de memória precisa ser avaliada e deve-se considerar a possibilidade de diferentes testes de memória produzirem resultados diferentes sobre os benefícios do treino administrado.
Van Erven e Janczura (2004) ressaltam o efeito do tipo de teste de memória no desempenho, pois as limitações da capacidade são específicas ao tipo de teste, podendo refletir a dificuldade da pessoa para integrar as informações (Light, 1991). A literatura relata que as falhas de memória dos idosos estão associadas a diversos fatores, uns mais do que outros, dependendo daqueles que são mais salientados pelo tipo de teste aplicado (Graf, 1990; Verhaeghen \& Marcoen, 1993).

Outro fator importante, quando se consideram os benefícios do treino cognitivo, é a influência da escolaridade dos participantes. O Brasil é um país em que, além da baixa escolaridade, há um grande número de analfabetos. Existem poucas pesquisas sobre o desempenho de analfabetos em avaliações neuropsicológicas, pois o uso desse grupo como medida de desempenho ainda representa uma tarefa incomum (Foss, Vale \& Speciali, 2005). Esses autores destacam a necessidade de estudos sobre o desempenho neuropsicológico que supram falhas e forneçam dados sobre fatores e variações nessa população.

Esta pesquisa contribui na investigação sobre os efeitos de diferentes treinos cognitivos no desempenho de memória e medidas neuropsicológicas em idosos institucionalizados, e examina a relação entre a escolaridade dos idosos e a intervenção proposta. Além disso, é objetivo deste trabalho oferecer aos profissionais envolvidos com essa população uma ferramenta que poderá contribuir para a reabilitação de idosos institucionalizados.

\section{Método}

Participantes

Os participantes eram oriundos de uma instituição asilar do Distrito Federal. A amostra excluiu os idosos acamados, com problemas psiquiátricos, hospitalizados, com patologias neuropsicológicas, e aqueles que evidenciassem comprometimento na Escala de Depressão Geriátrica (EDG) e na Avaliação Rápida das Funções Cognitivas (ARFC). A avaliação dos potenciais participantes resultou em uma amostra de 105 idosos, dos quais apenas vinte e um completaram todas as etapas do treinamento. Somente os dados desses participantes foram considerados nas análises dos resultados. A média da idade dessa amostra foi 79,5 anos $(D P=6,67)$, incluindo $38 \%$ homens e $62 \%$ mulheres. Dezesseis idosos participaram efetivamente dos treinos e das avaliações pré e pós-treino, e cinco que não participaram dos treinos constituíram um grupo controle para o grupo de não-alfabetizados.

A caracterização dos participantes por condição experimental é a seguinte: o grupo alfabetizado e treino 
relacionado foi composto por 6 homens, com média de idade de 79,8 (DP=5,8); o grupo do treino menos relacionado incluiu 5 mulheres com média de idade de $82,5$ ( $D P=9,4)$. O grupo não-alfabetizado que participou do treino incluiu 5 mulheres $(M=78$, $D P=7,87)$. Participaram do grupo-controle 2 homens e 3 mulheres $(M=77,8, \quad D P=4,76)$. Apesar de, inicialmente $(n=105)$, ter-se configurado os grupos de maneira semelhante quanto ao sexo dos participantes, a perda de participantes (p. ex., desistência, adoecimento, saída da instituição) ao longo das etapas experimentais determinou, aleatoriamente, que o grupo alfabetizado e treino relacionado incluísse somente homens. Entretanto, não há razões para acreditar que o sexo tenha sido uma fonte de variação significativa considerando a natureza das atividades, e a ausência de estudos apontando este fator nas condições testadas. Esta pesquisa foi aprovada pelo Comitê de Ética em Pesquisa da Faculdade de Saúde da Universidade de Brasília. Seguindo os critérios éticos, os idosos participaram de forma voluntária, mediante a assinatura de Termo de Consentimento Livre e Esclarecido por eles mesmos ou por seu representante legal, no caso dos não alfabetizados.

\section{Delineamento}

O efeito do treino e tipo de teste sobre a memória no grupo alfabetizado foi investigado aplicando-se um delineamento fatorial misto $2 \times 2 \times 2$ : tipo de treino (relacionado e menos relacionado), tipo de teste (recordação livre e reconhecimento) e momento da testagem (pré- e pós-treino). Os fatores tipo de teste e momento da testagem foram manipulados intrassujeitos, e o tipo de treino tratado entre sujeitos.

No grupo de participantes não-alfabetizados, comparou-se o efeito dos mesmos tipos de testes de memória e se o treino influenciaria o desempenho, configurando um delineamento fatorial 2 (com treino e sem treino) x 2 (recuperação livre e reconhecimento de figuras) x 2 momento da testagem (antes e depois do treino). $\mathrm{O}$ único fator manipulado entre sujeitos foi o treino, sendo os demais tratados intrassujeitos.

\section{Materiais}

Os materiais foram organizados em três grupos. $\mathrm{O}$ primeiro incluiu os instrumentos de triagem e parâmetros neuropsicológicos aplicados nas fases prée pós-treino. Foram administrados o Teste de Avaliação Rápida das Funções Cognitivas - ARFC (Gil, 2005; Gil, Toullat \& Pluchon, 1986) e a Escala de Depressão Geriátrica - EDG (Almeida \& Almeida, 1999).

O segundo grupo de materiais incluiu palavras e figuras utilizadas nos testes de memória. O Teste de
Recordação Livre continha 24 palavras retiradas das normas associativas de Janczura (1996), sendo 12 concretas e 12 abstratas. O Teste de Reconhecimento de Figuras incluía 75 figuras na fase de apresentação e 150 figuras na fase de teste, selecionadas das normas de Snodgrass e Vanderwart (1980).

O terceiro grupo de materiais ${ }^{1}$ consistiu dos estímulos utilizados nos treinos cognitivos que foram confeccionados pelos autores, observando normas já existentes na literatura ou em práticas executadas em reabilitação cognitiva. Um web designer auxiliou na confecção dos estímulos. Foram criadas 3 categorias de materiais, segundo a condição experimental: relacionado para os idosos alfabetizados, menos relacionado para os idosos alfabetizados e relacionado para os idosos não-alfabetizados. A descrição específica dos materiais é apresentada a seguir.

Liga pontos: consiste em um conjunto de nove figuras impressas em papel e organizadas em três grupos (cada um com três itens), que se diferenciam em função do grau de dificuldade, desde a mais fácil até a mais difícil. O Nível I inclui figuras simples, cujos contornos são tracejados; Nível II inclui figuras, cujos contornos estão delineados por números que vão de 1 a 30; e Nível III inclui figuras complexas, cujos contornos estão delineados por números que variam de 1 a 20 e letras que variam de $\mathrm{A}$ e $\mathrm{T}$.

Forma sequencial visual: consiste em um conjunto de 27 figuras projetadas por um DataShow, organizadas em três grupos (cada um com três histórias), que se diferenciaram em função do grau de dificuldade, desde o mais fácil até o mais difícil, a saber: Nível I incluiu duas figuras para cada história, totalizando seis figuras; Nível II incluiu três figuras para cada história, totalizando nove figuras; Nível III incluiu quatro figuras para cada história, totalizando dezesseis figuras; além de folha de respostas enumeradas de 1 a 9 e com espaços correspondentes para as respostas da ordem correta das histórias apresentadas.

Lista de palavras: 96 palavras selecionadas das normas de concretude de Janczura, Castilho, Rocha, Van Erven e Huang (2007) pertencentes a quatro categorias (alimento, objetos, profissões e jogos/hobbies) e relacionadas ao próprio ambiente do idoso, sendo 24 palavras de cada categoria; além da folha de respostas.

Aprendizagem associativa: consiste de 30 frases organizadas em três grupos (cada um com dez frases) que se diferenciaram em função do grau de dificuldade e do número de elementos constituintes, desde o mais fácil até o mais difícil, a saber: Nível I, Nível II e Nível

\footnotetext{
${ }^{1}$ Esses materiais estão disponíveis com os autores.
} 
III, que continham frases com três, quatro e cinco palavras, respectivamente; além da folha de respostas.

Categoria de memória: incluiu as 96 palavras utilizadas no treino de listas de palavras e mais 48 palavras para cada categoria, que funcionaram como palavras distraidoras. A folha de respostas incluía, ao lado de cada palavra, as alternativas "Sim" e "Não" para serem assinaladas.

Memória de imagens: 80 imagens, sendo 40 destas utilizadas na fase de treino e outras 40 acrescentadas na fase de teste que funcionaram como distraidoras. As figuras foram selecionadas do IAPS - International Affective Picture System, em função das dimensões prazer, alerta e dominância (Lang, Bradley \& Cuthbert, 1999). Além disso, a folha de resposta estava enumerada de 1 a 80 e ao lado de cada número havia as opções "Sim" e "Não".

Memória de histórias: consiste em um conjunto de três tipos de materiais, sendo o primeiro composto por duas histórias (uma relacionada e outra não relacionada à rotina do idoso). Para a história com conteúdo não relacionado à rotina do idoso foi utilizada a Escala de Memória Weschler (EMW), Subteste II - Memória Lógica e, para a história com conteúdo relacionado à rotina do idoso, fez-se uma adaptação da respectiva história, contemplando o ambiente dos idosos; o segundo material foi composto por uma folha com 16 operações aritméticas e o terceiro por uma folha com 2 figuras para o jogo dos 7 erros, sendo as figuras retiradas das normas de Snodgrass e Vanderwart (1980). Além disso, havia uma folha de respostas com os espaços correspondentes à tarefa sobre as histórias, operações aritméticas e jogo dos 7 erros.

\section{Procedimentos}

Avaliação neuropsicológica: $\mathrm{O}$ procedimento da avaliação neuropsicológica (ARFC e EDG) foi aplicado oralmente no pré-treino e pós-treino no período de uma sessão com, aproximadamente, 60 minutos e de forma individual.

Testes de memória: Os testes foram aplicados após a avaliação neuropsicológica, para cada um dos 3 grupos, utilizando o aplicativo SuperLab 4.0 (Cedrus Corporation, 2004). Os participantes responderam inicialmente ao Teste de Recordação Livre de Palavras e ao Teste de Reconhecimento de Figura.

A mesma versão do teste de memória foi aplicada antes e após o treino. A fim de minimizar a possibilidade de efeitos do treino, os seguintes cuidados foram tomados: randomização das palavras e figuras, assim como na ordem de aplicação dos testes em ambos os momentos. Além disso, considerou-se que as tarefas verbais e visuais realizadas durante o treinamento, que se estendeu por quase quatro semanas, tenham reduzido substancialmente a possibilidade de efeitos da aprendizagem dos testes de memória.

Os idosos não-alfabetizados realizaram os mesmos testes aplicados aos alfabetizados, havendo uma alteração de procedimento em relação ao teste de Recordação Livre de Palavras. Isto é, as palavras foram lidas em voz alta pelo(a) examinador(a), seguindo uma ordem de apresentação aleatória e na mesma velocidade das demais condições, e os idosos as repetiam em voz alta. Com relação ao Teste de Reconhecimento de Figuras não houve alteração de procedimento. Os grupos foram avaliados em momentos diferentes, antes e após os treinos cognitivos.

Treino cognitivo: Para a execução do treino, desenvolvemos duas versões, denominadas de versão mais relacionada à rotina do idoso e versão menos relacionada. Uma análise preliminar do ambiente institucional foi realizada para identificar quais elementos estariam mais e menos relacionados à respectiva rotina.

O treino cognitivo foi realizado duas vezes por semana, com duração de 1 hora, sendo monitorados a cada sessão a participação, o desempenho individual e as respostas preenchidas pelos idosos (somente para o grupo alfabetizado), mediante observação direta, com uso de anotações. Monitores treinados auxiliaram na aplicação das três categorias de treino cognitivo: relacionado à rotina do idoso, menos relacionado à rotina do idoso e relacionado à rotina do idoso, mas adaptado para os idosos não-alfabetizados. Neste caso, as tarefas de leitura de estímulos e escrita de respostas eram executadas pela equipe de pesquisa.

Os procedimentos foram acompanhados de informações psicopedagógicas relacionadas aos objetivos e tarefas de cada sessão, como, por exemplo, a relação entre o treinamento e as atividades executadas diariamente pelos idosos na instituição.

Para um melhor entendimento do procedimento do treino cognitivo será apresentada uma breve descrição dos procedimentos específicos de cada atividade, destacando que elas possuíam níveis gradativos de dificuldade tanto entre cada atividade diária quanto entre as 7 sessões do treino cognitivo.

Na sessão denominada "liga pontos", a tarefa era conectar pontos para formar uma figura. $\mathrm{Na}$ sessão "forma sequência visual" o idoso deveria colocar em ordem figuras apresentadas, formando uma história de acordo com o símbolo apresentado junto a cada figura. $\mathrm{Na}$ sessão "lista de palavras", a tarefa do idoso era, após ouvir uma lista de palavras, escrever todas as palavras que conseguisse lembrar. 
$\mathrm{Na}$ "aprendizagem associativa" a tarefa era escutar uma lista com frases e posteriormente escrever as frases lembradas. Na sessão "categoria de memória", a tarefa do idoso era escutar palavras de dadas categorias $\mathrm{e}$, posteriormente, reconhecer quais delas haviam sido apresentadas anteriormente e quais eram novas.

$\mathrm{Na}$ "memória de imagens" a tarefa era prestar atenção em imagens apresentadas por meio de slides e posteriormente reconhecer quais delas haviam sido apresentadas anteriormente e quais eram novas. $\mathrm{Na}$ última sessão, intitulada "memória de histórias", os idosos ouviam histórias e, logo após, tentavam lembrar o maior número possível de palavras da história, tanto de forma imediata (logo após a apresentação) como de forma tardia (após a execução de uma atividade distraidora).

\section{Resultados}

Os resultados dos grupos alfabetizados e não alfabetizados foram analisados separadamente porque, apesar de o treino do grupo não-alfabetizado ter sido semelhante ao do grupo alfabetizado, as adaptações do procedimento para o grupo não-alfabetizado tornaram inadequadas comparações entre tratamentos. Somente foram incluídos nas análises aqueles participantes que tiveram desempenhos equivalentes nas variáveis dependentes antes do treino; e, em todas as análises estatísticas adotou-se um $\alpha=0,05$.

A Tabela 1 apresenta as medidas pré e pós-treino para cada condição experimental.

Tabela 1. Médias e desvios padrão pré e pós-treino para cada condição experimental

\begin{tabular}{|c|c|c|c|c|c|c|c|c|}
\hline \multirow[t]{3}{*}{ Alfabetizados } & \multicolumn{4}{|c|}{ Antes } & \multicolumn{4}{|c|}{ Depois } \\
\hline & \multicolumn{2}{|c|}{$\begin{array}{l}\text { Treino } \\
\text { relacionado }\end{array}$} & \multicolumn{2}{|c|}{$\begin{array}{l}\text { Treino menos } \\
\text { relacionado }\end{array}$} & \multicolumn{2}{|c|}{ Treino relacionado } & \multicolumn{2}{|c|}{$\begin{array}{l}\text { Treino menos } \\
\text { relacionado }\end{array}$} \\
\hline & $\mathrm{M}$ & $\mathrm{DP}$ & $\mathrm{M}$ & $\mathrm{DP}$ & $\mathrm{M}$ & DP & $\mathrm{M}$ & DP \\
\hline ARFC & 34,3 & 4,47 & 33 & 8,43 & 42,9 & 4,44 & 35 & 8,17 \\
\hline EDG & 6,16 & 3,56 & 12 & 9,08 & 4,5 & 4,5 & 10,4 & 8,17 \\
\hline Recordação livre & 1,5 & 1,5 & 2,8 & 1,3 & 2,8 & 1,9 & 4,0 & 2,23 \\
\hline Hits & 45,3 & 20,09 & 56,6 & 15,63 & 42,6 & 15,7 & 63 & 16,04 \\
\hline Acertos & 60 & 12,04 & 46,8 & 28,39 & 62,6 & 12,11 & 50 & 26,26 \\
\hline Erros & 29,8 & 20,11 & 18,4 & 16,05 & 31,8 & 15,79 & 12,4 & 16,20 \\
\hline Alarme falso & 14,8 & 11,82 & 28,2 & 28,79 & 12,8 & 12,85 & 25,2 & 26,09 \\
\hline \multirow[t]{3}{*}{ Não alfabetizados } & \multicolumn{4}{|c|}{ Antes } & \multicolumn{4}{|c|}{ Depois } \\
\hline & \multicolumn{2}{|c|}{$\begin{array}{l}\text { Treino } \\
\text { relacionado }\end{array}$} & \multicolumn{2}{|c|}{$\begin{array}{l}\text { Ausência de } \\
\text { treino }\end{array}$} & \multicolumn{2}{|c|}{$\begin{array}{c}\text { Treino } \\
\text { relacionado }\end{array}$} & \multicolumn{2}{|c|}{ Ausência de treino } \\
\hline & $\mathrm{M}$ & DP & $\mathrm{M}$ & DP & $\mathrm{M}$ & $\mathrm{DP}$ & $\mathrm{M}$ & DP \\
\hline ARFC & 26,3 & 5,70 & 20,9 & 8,18 & 32,5 & 4,79 & 21,4 & 5,54 \\
\hline EDG & 6,2 & 4,54 & 9,8 & 7,12 & 6,4 & 3,71 & 10,6 & 7,33 \\
\hline Recordação livre & 1,8 & 1,3 & 0,8 & 0,8 & 2,8 & 2,28 & 1,0 & 1,30 \\
\hline Hits & 54,2 & 21,04 & 41 & 10,51 & 51,6 & 8,26 & 35,2 & 12,89 \\
\hline Acertos & 43,6 & 30,33 & 45,6 & 19,98 & 49,4 & 25,92 & 59,8 & 14,34 \\
\hline Erros & 20,8 & 21,04 & 35 & 12,06 & 23,4 & 8,26 & 39,6 & 12,58 \\
\hline Alarmes falsos & 31,4 & 30,33 & 28,4 & 21,33 & 25,6 & 25,92 & 15,4 & 14,25 \\
\hline
\end{tabular}

Legenda: $\mathrm{M}=$ média; DP=Desvio padrão; $\mathrm{ARFC}=$ Avaliação Rápida das Funções Cognitivas; EDG=Escala de Depressão Geriátrica.

\section{Grupos alfabetizados}

Foram realizadas análises para verificar se havia diferenças significativas entre os grupos alfabetizados com relação às variáveis dependentes e de controle antes do treino de memória. Análises de variância (ANOVAS) demonstraram não haver diferenças significativas pré-treino. Além disso, a análise mostrou que o número de sessões realizadas pelos grupos alfabetizados do Treino 1 (relacionado) versus Treino 2 (menos relacionado) também não foram significativamente diferentes. No que se refere ao ARFC, uma análise da variância mista para dois fatores foi calculada para avaliar o efeito do tipo de treino como fator entre grupos e o momento da avaliação (antes e pós-treino) como fator intragrupos. A ANOVA evidenciou como fontes significativas o momento do treino $[\mathrm{F}(1,9)=16,641, \mathrm{MS}=153,73$, $\mathrm{p}=0,003]$, e a interação entre o momento da avaliação e tipo de treino, $\mathrm{F}(1,9)=6,439$, $\mathrm{MS}=59,100, \mathrm{p}=0,032$. Ou seja, o ARFC após o treino foi superior $(M=39,3$, $D P=7,32)$ a antes do treino $(M=33,7, D P=6,2)$.

O Teste $\mathrm{T}$, com correção para Bonferroni, indicou que, na interação entre o tipo de treino e o momento da avaliação, a única diferença significativa 
observada foi o ARFC antes e após no grupo que recebeu o treino 1 .

No Teste de Recordação Livre a lembrança aumentou de maneira significativa após o treino, $\mathrm{F}=(1$, 9) $=7,117, \mathrm{MS}=8,752, \mathrm{p}=0,02$. A média de palavras recordadas antes do treino foi 2,09 e, após, 3,36, ou seja, um aumento de $8,7 \%$ para $14 \%$ do total de palavras lembradas. Nenhuma outra fonte de variação foi significativa.

No reconhecimento de figuras, o participante era solicitado a discriminar entre as figuras que haviam sido vistas na fase de estudo outras que não haviam sido apresentadas. Os dados foram tabulados em razão do tipo de item, podendo ser alvos ou distraidores. Posteriormente, os acertos para cada item foram transformados em hits, os acertos aos distraidores em acertos, os erros aos distraidores em falsos alarmes, e os erros aos alvos em erros (Banks, 1970 como citado em Lockart, 2000). A ANOVA não detectou diferenças significativas neste teste de memória. Discriminadamente, verificou-se que os hits tiveram uma porcentagem média de reconhecimento dos alvos alta, tanto antes $(67 \%)$ quanto após o treino (69\%); para os acertos a porcentagem média de reconhecimento dos distraidores também foi alta antes $(72 \%)$ e após o treino (76\%); a porcentagem média de erros foi baixa antes $(33 \%)$ e após $(30 \%)$; e, finalmente, os alarmes falsos obtiveram uma porcentagem média de $20 \%$ nos dois momentos.

Um teste para amostras pareadas detectou uma redução significativa de 9 pontos iniciais para 7,18 após o treino na Escala de Depressão Geriátrica (EDG), $\mathrm{t}(10)=2,557, \quad \mathrm{p}=0,029$. Analisando os grupos separadamente, verificou-se que a média do grupo que realizou o treino relacionado à rotina do idoso caiu de 6,16 para 4,5 e o grupo que realizou o treino menos relacionado a rotina reduziu de 12 para 10,4.

\section{Grupos não-alfabetizados}

Testes $\mathrm{t}$ para amostras independentes foram calculados para verificar se o grupo-controle e o grupo experimental eram diferentes antes do treino nas cinco medidas coletadas: avaliação rápida das funções cognitivas, recordação livre, bits, acertos, erros e alarmes falsos. Nenhuma diferença estatisticamente significativa entre os grupos foi encontrada.

Uma ANOVA mista para dois fatores foi calculada para avaliar o efeito do treino no ARFC. O tipo de grupo (controle versus experimental) foi tratado como fator entre grupos, e o momento da avaliação (antes versus depois do treino) como medida repetida. Identificou-se uma influência significativa quanto à participação no treino, $\mathrm{F}=(1,8)=6,233$, MS $=68,450$, $\mathrm{p}=0,037$. Os participantes não-alfabetizados aumentaram o ARFC após o treino; a média geral subiu de 23,6 para 27. No entanto, ao analisar separadamente os grupos verificou-se que a média dos participantes não-alfabetizados subiu de 26,3 para 32,5 e o grupo-controle permaneceu estável em torno de 21 pontos.

Com relação aos testes de Recordação Livre e Reconhecimento de Figuras, nenhum efeito significativo foi detectado, assim como na Escala de Depressão Geriátrica (EDG).

\section{Discussão}

Esse estudo teve como objetivo investigar a influência de diferentes treinos cognitivos de memória em idosos institucionalizados. A escolha por idosos institucionalizados justificou-se pela carência de trabalhos com essa população e para identificar alguns aspectos que revelem sua condição.

Três questões merecem consideração, inicialmente. A primeira se relaciona a possíveis diferenças entre treinos mais e menos relacionados à rotina de idosos institucionalizados. O teste ARFC foi sensível ao efeito do tipo de treino nos grupos alfabetizados, mais especificamente no treino mais relacionado à rotina do idoso. Esse resultado é consistente com o efeito de autorreferência, no qual as pessoas tendem a recordar mais fatos relativos a elas mesmas (Anderson, 2004).

A segunda questão se relaciona à inserção do treino cognitivo relacionado à rotina dos idosos quando esses são institucionalizados e nãoalfabetizados. O treino também beneficiou esse grupo. Os dados do ARFC, coletados após os treinos, demonstraram que os idosos do grupo experimental obtiveram ganhos significativos ante o grupo-controle. As seguintes funções do ARFC foram mais beneficiadas pelo treino: orientação, atenção e memória, raciocínio e julgamento e compreensão. Este resultado sugere que o treino cognitivo de memória pode ser estendido para essa parcela da população idosa institucionalizada.

A última questão trata da relação entre o tipo de teste de memória - recordação livre de palavras e reconhecimento de figuras - e a influência do treino específico considerando cada grupo de idosos.

No Teste de Recordação Livre observou-se um aumento geral na taxa de lembrança, independentemente do tipo de treino para o grupo alfabetizado. Ou seja, o treino mais relacionado à rotina do idoso não produziu taxas superiores, conforme era esperado. Uma hipótese para este resultado se relaciona ao tamanho da amostra, visto que "a probabilidade de uma amostra grande fornecer dados que reflitam 
exatamente o valor real da população aumenta com o aumento do tamanho da amostra" (Cozby, 2009, p. 147). No caso, as amostras testadas eram pequenas, diminuindo a chance de se detectar o efeito esperado, e sabe-se que, para a obtenção de resultados mais robustos, são necessários estudos que envolvam mais participantes e que realizem retestes de seguimento, buscando avaliar a duração da melhora (Mattos, Farina, Oliveira \& Yassuda, 2012).

Uma possibilidade razoável para a influência do treino no Teste de Recordação Livre apenas nos grupos alfabetizados se relaciona à fluência verbal uma das funções cognitivas contempladas pelo ARFC. A fluência verbal pode ter influenciado e beneficiado o desempenho no Teste de Memória com material verbal. É importante lembrar, como afirmam Foss, Vale e Speciali (2005), que

- fato de indivíduos de outras culturas e
analfabetos apresentarem desempenhos inferiores,
em testes neuropsicológicos, não necessariamente
reflete apenas perdas nas habilidades cognitivas
avaliadas, mas, também, remonta a falta de
testes adequados para medir analfabetos e
indivíduos pertencentes a culturas diferentes. (p.
126)

No Teste de Reconhecimento de Figuras, em nenhum grupo verificou-se a influência do treino no desempenho da memória. Acredita-se que esse teste não foi sensível aos treinos por ter produzido um efeito teto, ou seja, o teste foi demasiadamente fácil para os participantes. Avaliou-se, inicialmente, que a quantidade de figuras do teste seria suficiente considerando o nível de comprometimento apresentado pelos participantes no pré-teste. Todavia, os resultados evidenciaram que o número de estímulos pictóricos deverá ser aumentado significativamente em estudos futuros.

Outro ponto que merece destaque são os resultados da Escala de Depressão Geriátrica (EDG). Os escores mostraram que para os idosos alfabetizados houve um efeito positivo independentemente do tipo de treino administrado. Isto é, o fato de eles realizarem atividades proporcionadas pelo treino cognitivo, por mudança nas atividades diárias, ou, ainda, pela presença de novas pessoas no ambiente acompanhando, conversando, interagindo socialmente, produziu modificações no humor.

Os efeitos positivos do treino relatados neste estudo são consistentes com a literatura da área (p. ex., Balota, Dolan \& Duchek, 2000; Lautenschalager, 2002). O treino é importante na medida em que a capacidade de reserva cognitiva pode ser mobilizada e até mesmo melhorada, como é o caso da memória.

\section{Considerações finais}

É importante ressaltar que uma das principais limitações desta pesquisa deveu-se ao número de participantes. Porém, apesar dessa limitação, os resultados produzidos são encorajadores, sugerindo que o programa de treinamento proposto pode produzir benefícios para idosos institucionalizados. Trata-se de um grupo pouco estudado e com várias limitações associadas ao acesso e à execução de intervenções cognitivas. Este trabalho corroborou resultados anteriormente observados na literatura e, ao mesmo tempo, apontou a possibilidade desses idosos (alfabetizados ou não alfabetizados) serem beneficiados em aspectos cognitivos e emocionais, por meio de treinos cognitivos.

\section{Referências}

Almeida, O. P., \& Almeida, S. A. (1999). Confiabilidade da versão brasileira da escala de depressão em geriatria (GDS) versão reduzida. Arquivos de Neuropsiquiatria, 57, 421-426. Almeida, O. P., \& Forlenza, O. V.(1997). Depressão e demência no idoso: tratamento psicológico e farmacológico. São Paulo: Lemos.

Anderson, J. R. (2004). Psicologia cognitiva e suas implicações experimentais. Rio de Janeiro: LTC. Balota, D. A., Dolan, P. O., \& Duchek, J. M. (2000). Memory changes in health older adults. Em E. Tulving \& F. I. M. Craig (Eds.). The Oxford Handbook of Memory (pp. 395-410). Oxford: Oxford University Press.

Belleville, S. (2008). Cognitive training for persons with mild cognitive impairment. International Psychogeriatrics, 20(1), 57-66.

Belleville, S., Gilbert, B., Fontaine, F. Gagnon, L., Ménard, E. \& Gauthier, S. (2006). Improvement of episodic memory in persons with mild cognitive impairment and healthy older adults: evidence from a cognitive intervention program. Dementia and Geriatric Cognitive Disorders, 22(5-6), 486-499.

Belleville, S., Clément, F., Mellah, S., Gilbert, B., Fontaine. F., \& Gauthier, S. (2011). Trainingrelated brain plasticity in subjects at risk of developing Alzheimer's disease. Brain, 134(6), 1623-1634.

Cedrus Corporation (2004). SuperLab 4. CA, San Pedro, USA.

Cozby, P. C. (2009). Métodos de pesquisa em ciências do comportamento. São Paulo: Atlas.

D’Almeida, A., Pinna, D., Martins, F., Siebra, G., \& Moura, I. (2004). Reabilitação cognitiva de 
pacientes com lesão cerebral adquirida. CienteFico,4(1).

Folstein, M. F., Folstein, S. E., \& McHugh, P. R. (1975). Mini-mental state: a practical method for grading the cognitive states of patients for the clinician. Journal of Psychiatric Research, 12, 189-198.

Foss, M. P., Vale, F. A. C., \& Speciali, J. G. (2005). Influência da escolaridade na avaliação neuropsicológica de idosos. Arquivos de Neuropsiquiatria, 63, 119-126.

Gagnon, L. G., \& Belleville, S. (2012). Training of attentional control in mild cognitive impairment with executive deficits: Results from a doubleblind randomized controlled study. Neuropsychological Rebabilitation, 1,1-27.

Gil R. (2005). Neuropsicologia. (M. A. A. S, Doria, Trad.). São Paulo: Santos Liv. Ed.

Gil, R., Toullat, G., \& Pluchon, C. (1986). Une méthode d'evaluation rapide dês functions cognitives (ERFC). Son application à la démence senile de tupe Alzheimer. Semaine des Hôpitaux de París, 62, 2127-2133.

Graf, P. (1990). Life-span changes in implicit and explicit memory. Bulletin of the Psychonomic Society, 28(4), 353-358.

Gross, A. L., Rebok, G. W., Unverzagt, S. L., \& Brandt, J. (2011). Word list memory predicts everyday function and problem-solving in the elderly: results from the ACTIVE cognitive intervention trial. Aging, Neuropsychology, and Cognition, 18(2), 129-146.

Guerreiro, T., \& Caldas, C. P. (2001). Memória e demência: (re)conbecimento e cuidado. Rio de Janeiro: UERJ, UnATI.

Irigaray, T. Q., Schneider, R. H., \& Gomes, I. (2011). Efeitos de um treino cognitivo na qualidade de vida e no bem-estar psicológico. Psicologia Reflexão e Crítica, 24(4), 810-818.

Janczura, G. A. (1996). Normas associativas para 69 categorias semânticas. Psicologia: Teoria e Pesquisa, 12, 237-244.

Janczura, G. A., Castilho, G. M., Rocha, N. O., Erven, T. J. C. G. V., \& Huang, T. P. (2007). Normas de concretude para 909 palavras da língua portuguesa. Psicologia: Teoria e Pesquisa, 23, 195-204.

Kausler, D. H. (1991). Experimental psychology, cognition, and buman aging. New York: Springer-Verlag.

Kurz, A., Pohl, C., Ramsenthaler, M., \& Sorg, C. (2008). Cognitive rehabilitation in patients with mild cognitive impairment. International Journal of Geriatric Psychiatry, 23,1-6.

Lang, P. J., Bradley, M. M., \& Cuthbert, B. N. (1999). International affective picture system (IAPS): instruction manual and affective ratings. Technical report A-4, the center for research in psychophysiology. University of Florida.

Lautenschalager, N. T. (2002). É possível prevenir o desenvolvimento da demência? Revista Brasileira de Psiquiatria, 24(suppl), 7-22.

Lezak, M. (1995). Neuropsychological assessment. New York: Oxford University Press.

Light, L. L. (1991). Memory and aging: four hypotheses in search of data. Annual Review of Psychology, 42, 333-376.

Lockhart, R. S. (2000). Methods of memory research. Em E. Tulving \& F. I. M. Craig (Eds.). The Oxford handbook of memory (pp. 45-57). Oxford: Oxford University Press.

Luria, A. R. (1990). Desenvolvimento cognitivo. São Paulo: Ícone.

Mattos, M .R. O. D., Farina, N. S. M. J., Oliveira, A., \& Yassuda, M. A. (2012) Revisão: efeitos do treino de memória em idosos normais e em idosos com comprometimento cognitivo leve. Temas em Psicologia, 20(1), 235-245.

Montgomery, S. A., \& Asberg, M. A. (1979). A new depression rating scale designed to be sensitive to change. British Journal of Psychiatry, 134, 382-389.

Neely, A. S. (2000). Multifactorial memory training in normal aging: In search of memory improvement beyond the ordinary. Em R. D. Hill, L. Bäckman \& A. S. Neely (Eds.). Cognitive rebabilitation in old age (pp. 63-80). New York: Oxford Express.

Pavarini, S. C. I., Mediondo, M. S. Z., Barham, E. J., Varoto, V. A. G., \& Filizola, C. L. A. (2005). A arte de cuidar do idoso: gerontologia como profissão? Texto Contexto Enfermagem, 14, 398-402.

Regi, D. P., Junior, A. M., \& Castro, A. C. L. C. (2007). O idoso demenciado e seu impacto na Saúde Pública: estamos preparados? Metrocamp Pesquisa, 1, 18-36.

Salthouse, T. A. (1991). Mediation of adult age differences in cognition by reductions in working memory and speed of processing. Psychological Science, 2, 179-183.

Sheikh, J. I., \& Yesavage, J. A. (1986). Geriatric depression scale (GDS): recent evidence and development of a shorter version. Clinical Gerontology, 5, 165-173.

Snodgrass, J. G., \& Vanderwart, M. (1980). A standardized set of 260 pictures: norms for name agreement, image agreement, familiarity, and visual complexity. Journal of Experimental Psychology: Human, Perception and Performance, 6, 174-215.

Stuart-Hamilton, I. (2002). Psicologia del envejecimento. Madrid: Morata. 
Van Erven, T. J. C. G., \& Janczura, G. A. (2004). A memória de idosos em tarefas complexas. Psicologia: Teoria e Pesquisa, 20, 59-68.

Verhaeghen, P., \& Marcoen, A. (1993). Memory aging as a general phenomenon: episodic recall of older adults is a function of episodic recall of young adults. Psychology and Aging, 8, 380-388.

Wilson, B. A. (2009). Memory rehabilitation: integrating theory and practice. New York: The Guilford Press.

Yassuda, M. S. (2002). Memória e envelhecimento saudável. Em E. V. Freitas, A. N. Neri, F. A. X. Cançado, M. L. Gorzoni \& S. M. Rocha (Eds.).
Tratado de geriatria e gerontologia (pp. 914-920). Rio de Janeiro: Guanabara Koogan.

Yassuda, M. S., Batistoni, S. S. T., Fortes, A. G., \& Neri, A. L. (2006). Treino de memória no idoso saudável: benefícios e mecanismos. Psicologia Reflexão e Crítica, 19, 470-481.

Recebido em 10/06/2011

Reformulado em 28/08/2012

Aprovado em 30/01/2013

Sobre os autores:

Isabelle Patriciá Freitas Chariglione é mestre (2010) e doutoranda em Cognição e Neurociências do Programa de Pós-Graduação em Ciências do Comportamento da Universidade de Brasília. Atualmente encontra-se em estágio sanduíche no "Institut Universitaire de Gériatrie de Montréal" na Université de Montréal, Canadá. Especialista em Neuropsicologia pelo IPAF (2008), possui graduação em Licenciatura Plena (2004) e Formação em Psicologia (2005) pela Universidade Federal da Paraíba.

Gerson Américo Janczura é doutor em Psicologia Cognitiva Experimental - University of South Florida (1994), mestre em Psicologia pela Universidade de Brasilia (1986), e graduado em Psicologia pela Pontifícia Universidade Católica do Rio Grande do Sul (1982). Desenvolve pesquisas sobre a Memória Humana e Processos Cognitivos. É professor associado da UnB, responsável pelo Laboratório de Processos Cognitivos e pelo grupo de Pesquisa/CNPq: Memória e Comportamento.

Contato com os autores:

Universidade de Brasília

Asa Norte - CEP 70910-900 - Brasília/DF, Brasil 